\section{Change of heart on miR-21}

\section{By Joanne Kotz, Senior Editor}

A team led by researchers at The University of Texas Southwestern Medical Center at Dallas and miRagen Therapeutics Inc. has reported that blocking microRNA-21 has no effect on stress-induced cardiac dysfunction. The findings prompted miRagen to drop its miR21 program and pursue other miRNA targets for the indication. But Regulus Therapeutics Inc. is sticking by its miR-21 program in cardiac fibrosis based on its own positive preclinical data and maintaining that its program requires no course correction.

miRagen's interest in miR-21 dates to 2006, when Eric Olson and colleagues identified 21 miRNAs that were upregulated and 7 miRNAs that were downregulated in mouse models of cardiac stress compared with healthy controls. Of the upregulated miRNAs, miR-21 had the largest change in expression. ${ }^{1}$

Olson is chairman of molecular biology at UT Southwestern Medical Center and cofounder and chief scientific advisor of miRa-

still had levels of cardiac hypertrophy similar to those in mice given nontargeting oligonucleotides.

The results were reported in The Journal of Clinical Investigation.

"The combination of the genetic deletion experiments and treatment with potent anti-miRNAs convinced us, and we're not going to move forward with miR-21," said miRagen cofounder, president and CEO William Marshall.

\section{miRNA validation}

In contrast, Eric Marcusson, director of drug discovery at Regulus, said the JCI findings will have no impact on the company's preclinical development of anti-miR-21 therapeutics. The contrasting decisions result in part from different approaches for validating miRNA targets in animals.

At Regulus, Marcusson said genetic knockout experiments are a less important factor in decisions. "We know that knocking out a gene beginning in early development of an organism can have different effects from pharmacologically inhibiting a gene product in an adult animal," he said.

Instead, according to Marcusson, the company looks for targets in which inhibiting the miRNA across multiple disease models leads to a similar phenotype.

This has been the case for miR-21. Marcusson told SciBX that anti-miR-21 antisense oligonucleotides consistently produce a therapeutic benefit in preclinical models of both cardiac fibrosis and fibrosis in other organs.

miRagen places more emphasis on genetic validation of miRNA targets. "We believe that gen. Based on the findings, miRagen started preclinical programs evaluating a number of the miRNAs, including miR-21, as potential targets to treat heart failure.

A 2008 paper in Nature bolstered the case for the target. In it, a German research team and scientists at Regulus and Alnylam Pharmaceuticals Inc. reported that in a mouse model of stress-induced cardiac fibrosis, a chemically modified antisense oligonucleotide against miR-21 decreased cardiac expression of miR-21, cardiac fibrosis and cardiac hypertrophy compared with vehicle. ${ }^{2}$

Based on this evidence, Regulus established a preclinical program focused on miR-21 in 2009.

However, new findings stemming from miRagen's preclinical evaluation of the target run counter to those previous data. A team led by Olson and Eva van Rooij, cofounder and director of biology at miRagen, reported that modulating miR-21 produced no improvements in mice with cardiac fibrosis or hypertrophy. ${ }^{3}$

In the new work, the research team first looked at the effects of genetic ablation. In miR-21-deficient mice and wild-type controls, four different cardiac stresses each led to increases in cardiac hypertrophy or fibrosis.

In two mouse models of cardiac stress, a locked nucleic acid (LNA)modified antisense oligonucleotide against miR-21 decreased expression of the target in the heart to levels prior to stress, but animals genetic ablation is an important point in the overall package," said Marshall. "We get concerned if the genetic and pharmacological phenotypes don't match."

For other miRNA targets the company is pursuing, such as miR208 and miR-451, miRagen has seen congruence between genetic knockout and synthetic oligonucleotide inhibition experiments.

"Do I require genetic ablation to consider a target interesting? Not $100 \%$ of the time," said Marshall. Exceptions would include cases in which a family of miRNAs shares a common target-binding region. In such a situation, the knockout of one miRNA family member might be functionally compensated for by another during development, potentially obscuring a disease-modifying phenotype that would be seen with synthetic oligonucleotide inhibition in adult mice.

miR-21 has no obvious homologs, Marshall noted, and the closest potential homolog is not expressed in the heart and was not upregulated in the miR-21 knockout mouse.

\section{Therapeutic impact}

According to Marcusson, in addition to Regulus' doubts about the preclinical value of the genetic ablation data, it thinks the lack of therapeutic benefit seen in the JCI paper could be because "the 


\section{ANALYSIS}

\section{TARGETS \& MECHANISMS}

shorter LNA oligonucleotides used in the studies are not very potent inhibitors compared with longer anti-miRNA inhibitors."

Regulus has looked at LNA inhibitors, which are only eight bases in length, in multiple models. The company has "always found them to be less potent" than longer antisense oligonucleotides, Marcusson noted.

Marshall countered that miRagen has seen therapeutic benefit from LNA-modified oligonucleotide inhibition of many miRNA targets in heart disease and other cardiovascular and musculoskeletal indications.

He added that multiple independent groups have also published reports demonstrating excellent activity with LNA-modified oligonucleotides in a wide range of targets and indications.

In addition to continuing to investigate miR-21 as a target in heart fibrosis, Regulus has preclinical programs targeting miR-21 for fibrosis in other organs as well as in oncology.

Regulus holds patents covering miR-21 in heart disease and other indications. In June of this year, the company formed a partnership with sanofi-aventis Group to develop therapeutics for four miRNA targets in fibrosis, including miR-21. The agreement included an upfront investment of $\$ 25$ million with a potential of up to $\$ 750$ million.

Meanwhile, miRagen has turned to other miRNA targets for heart failure in which Marshall is more comfortable with the biology and preclinical data. The company's most advanced inhibitor targets miR-208, which regulates expression of a pathway involved in cardiac contraction during cardiac hypertrophy and fibrosis. ${ }^{4}$

The compound has shown efficacy in multiple animal models of heart failure and has been dosed in nonhuman primates. The company hopes to begin Phase I trials in early 2012.

"Because of the data we generated on the target," Marshall told SciBX, "miRagen abandoned patent filings on miR-21."

Kotz, J. SciBX 3(44); doi:10.1038/scibx.2010.1313

Published online Nov. 11, 2010

\section{REFERENCES}

1. van Rooij, E. et al. Proc. Natl. Acad. Sci. USA 103, 18255-18260 (2006)

2. Thum, T. et al. Nature 456, 980-984 (2008)

3. Patrick, D.M. et al. J. Clin. Invest.; published online Oct. 18, 2010; doi:10.1172/JCl43604

Contact: Eric N. Olson, The University of Texas Southwestern Medical Center at Dallas, Dallas, Texas

e-mail: eric.olson@utsouthwestern.edu

Contact: Eva van Rooij, miRagen Therapeutics Inc., Boulder, Colo. e-mail: evanrooij@miragenrx.com

4. van Rooij, E. et al. Science 316, 575-579 (2007)

\section{COMPANIES AND INSTITUTIONS MENTIONED}

Alnylam Pharmaceuticals Inc. (NASDAQ:ALNY), Cambridge, Mass. miRagen Therapeutics Inc., Boulder, Colo.

Regulus Therapeutics Inc., Carlsbad, Calif. sanofi-aventis Group (Euronext:SAN; NYSE:SNY), Paris, France The University of Texas Southwestern Medical Center at Dallas, Dallas, Texas 